\title{
The effect of the mother's heartbeat sound on physiological parameters and pain intensity after blood sampling in neonates in the intensive care unit: $A$ randomized controlled clinical trial
}

\section{ABSTRACT}

Aim: To examine the effect of the mother's heartbeat sound on physiological parameters and pain intensity after blood sampling in neonates in the intensive care unit.

Methods: A randomized controlled clinical trial was conducted on 60 neonates admitted to the intensive care unit. They were assigned to intervention and control groups ( $\mathrm{n}=30$ in each group). The intervention group listened to the mother's heartbeat sound, 10 minutes before up to 10 minutes after taking arterial blood samples. Pain intensity was measured every 10 minutes in 3 steps using the neonatal infant pain scale 10 minutes before the intervention, immediately after and 10 minutes after the intervention. At the same time, physiologic parameters including oxygen saturation, respiratory rate, heart rate and mean arterial blood pressure were recorded. Data were analyzed using descriptive and inferential statistics using SPSS.

Results: Listening to the mother's heartbeat sound did not influence on mean arterial pressure in the intervention group. However, it had significant medium to large effects on oxygen saturation and respiratory rate immediately after and 10 minutes after the intervention. Also, it had a large effect on heart rate immediately after the intervention $(\mathrm{P}<0.05)$. Also, significant medium to large effects of the intervention on pain intensity immediately after and 10 minutes after blood sampling were reported $(\mathrm{P}<0.05)$.

Conclusion: The mother's heartbeat sound can be used by nurses as a non-pharmacologic and safe intervention along with routine care in order to reduce suffering and pain in neonates undergoing invasive and painful procedures in the intensive care unit.

Keywords: intensive care unit, mother's heart beat, neonate, nursing care, pain, physiological parameters 


\section{Introduction}

Invasive procedures conducted by clinical nurses on neonates admitted to the intensive care unit including heel blood sampling, respiratory tract suction, peripheral vein path insertion, gastric tube and urinary catheter insertions are painful (Eghbalian and Shalchi 2014; Maroufi et al., 2015). Severity of pain can be from mild to severe in gastric tube insertion procedure (mild pain), heel blood sampling procedure (moderate pain) and spinal cord fluid sampling procedure (severe pain) (Levene, 2003). Full-term and preterm neonates can feel pain in response to annoying and painful stimuli and uncontrolled pain can impact on neural development. Therefore, painful procedures such as neonates' blood sampling cause psychological trauma and stress to neonates and even impacts on the brain structural development (Maroney, 2003; Sanders and Hall, 2018; World Health Organization, 2010). Painful and stressful stimuli can increase catecholamine release, heart rate, blood pressure and intracranial pressure (Claes et al., 2015; Imani and Moradi, 2016; Azarmnejad et al., 2015). In addition to the reduction of blood oxygen saturation and heart rate it causes acidosis and irregular breathing, increases blood sugar and stimulates the release of inflammatory hormones (Unesi et al., 2014, Maroufi et al., 2015). Pain relief during painful procedures can help with the prevention of unwanted physical and psychological effects (Saadatand et al., 2015). Therefore, one of the most important responsibilities of nurses in critical care units is to reduce neonates' suffering and pain during invasive procedures (McNair et al., 2013).

Pain often is controlled by medicines such as codeine and morphine for relieving severe pain that can cause respiratory depression, nausea, seizures and drug dependence (Alinejad-Naeini et al., 2014). Therefore, the role of non-pharmacological pain relieve strategies has been emphasized (Saadatand et al., 2015).

Non-pharmacological interventions and the use of alternative and complementary medicines such as nonnutritive sucking (NNS) with or without sucrose, breast milk or breastfeeding, swaddle, facilitated tucking, kangaroo care, music therapy and multisensory stimulation (SS) have been shown effective for the management of mild to moderate pain in neonates (Cignacco, et al., 2006). One of the potentially effective methods to relieve pain in neonates is to listen to the mother's heartbeat sound (Claes et al. 2017). Birth is a big change to neonates' lives and familiar phenomena can connect the neonate to the new environment (Nandhini et al., 2016). 
Hearing is one of the first senses that is developed in the fetus (Imani and Moradi, 2016). Also, the fetus has a significant capacity for voice recognition and auditory learning in the uterine environment (Rand, 2014). They receive sounds and respond to them with gestures from 26 to 28 weeks of pregnancy (Picciolini, 2014). One of the first auditory stimuli for fetuses is the mother's voice and heartbeat (Császár-Nagy, 2017), which are in the auditory range of 60-90 dB (Djordjevic, 2010). The mother's heartbeat, smell and warmth have soothing and calming effects on neonates (Nandhini et al., 2016; Babaei, Alhani and Khaleghipour, 2016). Previous studies have shown the effectiveness of the simultaneous use of the mother's voice and heartbeat sound in relieving pain in neonates (Azarmnejad et al., 2015; Doheny et al., 2012a) and improving their health-related indicators (Zimmerman et al., 2013; DeCasper and Prescott., 2009). However, there is a lack of knowledge of the effect of the mother's heartbeat sound alone on the improvement of physiological parameters during and after painful procedures in neonates admitted to neonatal intensive care units. Therefore, this study using an experiential design aimed to examine the effect of the mother's heartbeat sound on pain intensity and physiological parameters after blood sampling in neonates in the intensive care unit. The study hypothesis was that the mother's heartbeat sound reduced pain intensity and improved physiological parameters after blood sampling in neonates admitted to the intensive care unit.

\section{Methods}

A randomized controlled clinical trial was conducted in an urban area of Iran in 2014 in a sixmonth period. The research process has been shown using the CONSORT flow diagram (Figure 1).

\subsection{Sample and setting}

Subjects were 60 full-term neonates admitted to an intensive care unit in a teaching hospital. They were selected using a convenience sampling method and were randomly assigned to the intervention and control groups ( $\mathrm{n}=30$ in each group) through flipping coins.

Inclusion criteria were: full-term neonates with no hearing impairments based on the audiologist's diagnosis, not receiving analgesics at least three hours before and during blood sampling, absence of underlying diseases and not undergoing surgeries causing severe pain, absence of crippling diseases and anatomical abnormalities in limbs, and undergoing the arterial blood sampling procedure for laboratory and diagnostic purposes ordered by the physician. Those 
neonates who were undergoing ventilation and staff nurses failed to take blood samples in the first attempt were excluded.

\subsection{Data collection}

Data were gathered using the neonatal infant pain scale (NIPS) through recording videos from neonates before, during and after the arterial blood sampling procedure. The neonates' pain intensity was assessed and scored via the observation of the videos by a research assistant. NIPS is a behavioral scale for pain assessment in newborns, developed and used by Lawrence et al., in 1993. It can be used to measure pain in pre-term and full-term neonates until six weeks after birth (Hanson et al., 2010). It investigates crying, facial expressions, respiratory pattern, movements of hands and legs, and the state of arousal in newborns. Suraseranivongse et al., (2006) has confirmed reliability and validity of this scale. The validity and reliability of the Farsi version of this questionnaire has also been confirmed by Sarhangi et al. (2011) in Iran using the video recording method. They reported a interclass correlation coefficient (ICC) higher than 0.9. Also, the minimum correlation coefficient between inter-raters was reported 0.868. Additionally, a high correlation between the NIPS and the visual analog scale was reported $(r=0.949 ; p<0.001)$.

The mother's heartbeat sound for each neonate was recorded by the Cool Edit2000 software using the Summit Doppler Sonicade Series L350 (Summit Doppler Company ${ }^{\circledR}$, USA). Also, the neonates' heart rate, oxygen saturation, mean arterial pressure and respiratory rate were measured using the portable pulse oximeter device with 2\% calibration accuracy (Masimoset CA92614-V3, Poyandegan Rahe Saadat ${ }^{\circledR}$ ), which were registered on a researcher-made form by the research assistant. The weight of the neonates was measured using a digital scale (German vurf model, Beurer company ${ }^{\circledR}$ ) with the accuracy of 10 grams.

Moreover, the medical and demographic characteristics of the neonates including age, gender, medical diagnosis, duration of hospitalization, type of maternal delivery, Apgar score of one and five minutes after delivery, weight, maternal pregnancy duration (on a weekly basis) were found in the neonates' medical files and were registered on the researcher-made form by the main researcher.

Validity of the data collection tools was assessed through content validity by 10 faculty members who were experts in the fields of neonatal nursing care. Data were gathered 10 minutes before as the baseline, immediately after, and 10 minutes after the blood sampling procedure and were used for statistical analysis. 


\subsection{Intervention}

The accuracy of the neonates' hearing was assessed and confirmed using audio stimuli by a sound level meter via Oto-acoustic emission (OAE). Initial nursing care, diaper replacement and neonates' feeding were performed prior to the intervention. The arterial blood sampling procedure was video-taped from 10 minutes before, during and up to 10 minutes after the procedure. Next, the videos were observed and carefully assessed to detect changes in the neonates’ physiological criteria. Blood samples were taken by staff nurses using scalp vein needle number 23 in the intensive care unit. They were trained clinical nurses and were expert in performing this procedure and also followed the standard procedure for blood sampling.

The neonates in the intervention group were lying supine during taking blood samples and were in a separate room far from the intensive care unit to avoid noises. The mother's heartbeat sound was played inside the incubator using two small 200-watt speakers (SONY ${ }^{\circledR}$ ) placed on either side of the neonate's head, $20 \mathrm{~cm}$ from the neonate's ears with the sound threshold about $50 \mathrm{~dB}$ set by an audiologist. The sound was played 10 minutes before blood sampling and was continued until 10 minutes after it. The intervention duration was approximately thirty minutes on average.

In the control group, neonates received routine care in the intensive care unit except for listening to the mother's voice. In other words, the speaker was placed to the incubator for neonates in the control groups, but no sound was played.

To prevent bias during the data collection, the sound of the videos was cut off when a research assistant observed the videos and filled out the NIPS and read the physiologic parameters on the monitors, because she had to be blind to the group assignments. Also, the statistician was blind to the group assignments during data analysis.

\subsection{Data analysis}

Descriptive and inferential statistics were used for data analysis via SPSS version 19. Demographic and medical variables were presented using frequency, percentage, mean and standard deviation. The Kolmogorov-Smirnov test was used to assess the normal distribution of data. The independent t-test, the Fisher's exact tests and ANOVA tests were used for between groups and within group comparisons of demographic and medical variables and physiological parameters. The Mann-Whitney U test and the Friedman test were used for between groups and within group comparisons of pain intensity. $\mathrm{P}<0.05$ was considered statistically significant. 


\subsection{Ethical considerations}

Informed consent was obtained from the neonates' parents after explaining the process and aim of the study. Also, permission to record videos of the neonates was achieved with the consideration of confidentiality of the neonate's information and recorded videos. The neonates' parents were ensured of the voluntary nature of participation in the study and the possibility of withdrawal from it without having negative effects on the neonates' treatment and care process. Those parents who accepted to participate in the study signed the informed consent form. Since taking blood samples were ordered by the physician on the basis of laboratory and diagnostic purposes and the procedure was performed by the expert staff nurse who followed the standard guideline for blood sampling, no risk of harm for neonates was present.

\section{Results}

All 60 neonates assessed were eligible for inclusion and were recruited into the study. The neonates' parents agreed to participate and fully collaborated throughout the study process. The neonates had no statistically significant differences in terms of medical and demographic characteristics, and therefore were homogeneous (Table 1). Half of the neonates were male and half were female. Giving birth by the cesarean section and normal vaginal delivery between the groups was similar.

The comparison of the groups in terms of physiological parameters and the pain intensity were described as follows:

\subsection{Heart rate}

There was no statistically significant difference between the groups by intergroup comparisons at the baseline. While there was a statistically significant difference in heart rate between the groups immediately after the intervention $(\mathrm{P}=0.001)$ indicating its large effect in the intervention group $(\mathrm{d}=1.56)$, the groups were homogeneous 10 minutes after the intervention. Therefore, it was found that the intervention had no long lasting effect $(\mathrm{P}=0.46)$. Also, intra-group comparisons showed statistically significant differences between the control and intervention groups at three data collection times $(\mathrm{P}<0.05)$ (Table 2$)$.

\subsection{Respiratory rate}

There was no statistically significant difference between the groups at the baseline in respiratory rate, but differences were observed between the groups immediately after the intervention 
$(\mathrm{P}=0.01)$ and 10 minutes after the intervention $(\mathrm{P}=0.001)$. The effect of the intervention was reported from medium ( $\mathrm{d}=0.64)$ to large $(\mathrm{d}=0.86)$. Intra-group comparisons showed statistically significant differences between the control and intervention groups at three measurement times $(\mathrm{P}<0.05)$ (Table 2).

\subsection{Oxygen saturation}

No statistically significant difference between the groups in oxygen saturation at the base line was found. However, differences between the groups immediately after the intervention $(\mathrm{P}=0.001)$ and 10 minutes after the intervention $(\mathrm{P}=0.001)$ were statistically significant indicating the large effect of the intervention at both measurement times ( $d=1.16$ and $d=0.98$, respectively). Intra-group comparisons showed statistically significant differences between the control and intervention groups at three measurement times $(\mathrm{P}<0.05)$ (Table 2).

\subsection{Mean arterial pressure}

There were no statistically significant differences between the groups in mean arterial pressure at the baseline as well as immediately after the intervention and 10 minutes after the intervention $(\mathrm{P}>0.05)$. Also, intra-group comparisons did not show statistically significant differences in the control and intervention groups at three data collection times $(\mathrm{P}>0.05)$ (Table 2).

\subsection{Pain intensity}

There was no significant difference between the groups at the baseline in pain intensity. Statistically significant differences between the groups immediately after the intervention $(\mathrm{P}=0.004)$ and 10 minutes after the intervention $(\mathrm{P}=0.001)$ were reported indicating the medium $(d=0.77)$ to large effect ( $d=1.29)$ of the intervention. Intra-group comparisons showed statistically significant differences between the control and intervention groups at three measurement times $(\mathrm{P}=0.001)$ (Table 3).

\section{Discussion}

This clinical controlled trial examined the effect of the mother's heartbeat sound on physiological parameters and pain intensity after blood sampling in neonates in the intensive care unit. Our findings showed that listening to the mother's heartbeat sound did not affect mean arterial pressure, but it had medium to large effects on oxygen saturation and respiratory rate 
immediately after and 10 minutes after the intervention, as well as had a large effect on heart rate immediately after the intervention. Also, statistically significant medium to large effects of the intervention on pain intensity immediately after and 10 minutes after it were reported.

No exactly similar study in terms of the data collection process and outcome measures was found to be used for comparison with our findings. However, previous studies in general support the effectiveness of listening to the mother's heartbeat and voice on some physiological parameters and responses in neonates. For instance, DeCasper's (1983) and DeCasper and Prescott (2009) found that the recorded sound of the mother's heartbeat increased sucking in neonates compared to listing to non-familiar female sounds. Also, the Charpak (2005) and Zimmerman (2013) studies showed that the mother's heartbeat sound caused weight gain in neonates. Yildirim (2015) in the study of the effect of listening to the mother's heartbeat sound and uterine sound on the depth of anesthesia in children confirmed that the mother's heartbeat sound reduced blood pressure and heart rate compared to neonates listening to the environment sound. The Kurihara Hiroyuki et al.'s study (1996) showed that the mother's heartbeat sound reduced pain related to the heel blood sampling procedure in 131 neonates listening to the mother's heartbeat sound, music, and control. Also, these neonates showed less facial changes, less crying and lower cortisol and dehydroepiandrosterone sulfate levels in the blood than the other two groups. The Doheny's (2012a) study showed premature neonates' heartbeat and respiratory problems decreased in response to the mother's voice recordings and heartbeat sound. Hoe-Ludington (2006) reported that a combination of the mother's voice and heartbeat sound during kangaroo care led to normalizing body temperature, breathing and heart rate in neonates. Doheny (2012b) in a case report of a premature neonate who was exposed to the mother's recorded voice reported the stability of heart rate and prevention of apnea and bradycardia. The Rand's (2014) study showed a decrease in heart rate in the first month of life in premature neonates due to listening to maternal sounds.

The strength of this study lies in data collection immediately after and 10 minutes after the intervention leading to the assessment of both the short-term and long-term effects of the mother's heartbeat sound. As a limitation, it was impossible to eliminate all noises in the research zone that might have affected the study outcome, but sound levels were monitored by a sound 
meter throughout the intervention to prevent reaching sound in an unsafe decibel level to the neonates' ears, when it was combined with the intervention sound.

\section{Conclusion}

The present study showed the short-term and long-term effects of the mother's heartbeat sound on the reduction of pain intensity and physiological parameters during the arterial blood sampling procedure. Since the presence of the mother at the neonate's bedside in case of hospitalization in the intensive care unit is not always possible, the use of the mother's heartbeat sound by nurses for reducing pain and sufferings in neonates during painful and invasive procedures is suggested. Also, more clinical trails are needed to assess the effect of the mother's heartbeat sound on neonates' physiological and psychological parameters and compare it with other alternative and complementary medicines methods in intensive care units.

\section{Acknowledgments}

The authors would like to thank the neonates' parents as well as the collaboration of staff nurses in the intensive care unit with this research project.

\section{References}

Alinejad-Naeini, M., Mohagheghi, P., Peyrovi, H., Mehran, A., 2014. The effect of facilitated tucking during endotracheal suctioning on procedural pain in preterm neonates: a randomized controlled crossover study. Glob. J. Health Sci. 6(4), 278-284.

Azarmnejad, E., Sarhangi, F., Javadi, M., Rejeh, N. 2015. The Effect of Mother's Voice on Arterial Blood Sampling Induced Pain in Neonates Hospitalized in Neonate Intensive Care Unit. Glob J Health Sci.7(6),198-204.

Babaei, K., Alhani, F., Khaleghipour, M. 2016. Effect of Mother's Voice on Postoperative Pain Pediatric in Tonsillectomy Surgery. JPEN. 3 (2) 51-56.

Charpak, N., Ruiz, J.G., Zupan, J., Cattaneo, A., Figueroa, Z., Tessier, R., et al., 2005. Kangaroo mother care: 25 years after. Acta Paediatrica. 94(5),514-522.

Cignacco, E., Hamers, J P.H., Stoffel,L., Lingen,R. A. v., Gessler, P., McDougall, J., Nelle, M. 2006. The efficacy of non-pharmacological interventions in the management of procedural pain in preterm and term neonates A systematic literature review. Eur J Pain. 11(2):139-152.

Claes, S., et al. 2017. Hugsy: A Comforting Solution for Preterm Neonates Designed to Enhance Parent-Child Bonding. Applications, Systems and Engineering Technologies (CHASE), 
IEEE/ACM International Conference on. 2017. IEEE. 177-184. Available from the URL: https://ieeexplore.ieee.org/document/8010631 (Acessed on Juli 8, 2020)

Császár-Nagy, N., Bókkon, I., 2017. Mother-newborn separation at birth in hospitals: A possible risk for neurodevelopmental disorders? Neurosci Biobehav Rev. 84,337-351.

DeCasper, A.J., Prescott, P. 2009. Lateralized processes constrain auditory reinforcement in human newborns. Hear Res. 255(1-2),135-141.

Djordjevic, D. 2010. Premature Born Infant's Reaction to the Mother's Voice in Comparison to their Reaction to Music-Effect on Heartbeat and Heartbeat Variability,135-141.

Doheny, L., Hurwitz, Sh., Insoft, R., Ringer, S., Lahav, A. 2012a. Exposure to biological maternal sounds improves cardiorespiratory regulation in extremely preterm infants, J Matern Fetal Neonatal Med. 25(9), 1591-1594.

Doheny, L., Morey, J.A., Ringer, S.A., Lahav, A. 2012b. Reduced frequency of apnea and bradycardia episodes caused by exposure to biological maternal sounds. Pediatr Int. 54(2),e1-3.

Eghbalian, F., Shalchi, Z. 2014. Comparing the analgesic effects of glucose, breast feeding, acetaminophen and sterile water on pain relief in neonates. J Urmia Univ Med Sci. 25 (7), 578585.

Kurihara H., Chiba H., Shimizu Y., et al. 1996. Behavioral and adrenocortical responses to stress in neonates and the stabilizing effects of maternal heartbeat on them. Early Hum Dev. 46(12):117-127.

Ludington-Hoe S., Lewis T., Morgan K., Cong X., Anderson L., Reese S. 2006. Breast and infant temperatures with twins during shared kangaroo care. J. Ostet. Gynecol. Neonatal Nurs. 35(2), 223-231.

Hanson D, Hall W, Mills LL, Au S, Bhagat R, Hernandez M, et al. 2010. Comparison of distress and pain in infants randomized to groups receiving standard versus multiple immunizations. Infant Behavior and Development, 33(3), 289-96.

Imani, A., Moradi, F. 2016. Comparing the Effect of 30, 20 and 50 pecent Oral Sucrose on Reducing Behavioral Pain Responses Due to Hepatitis B Vaccine Injection in Neonates. J Urmia Univ Med Sci. 13 (12),1022-1028.

Marofi, M., Nikobakht, F., Badiee, Z., Golchin, M. 2015. The effect of melody on the physiological responses of heel sticks pain in neonates. IUMS, 20(3), 405-408.

Maroney, D.I. 2003. Recognizing the potential effect of stress and trauma on premature infants in the NICU: how are outcomes affected?. J Perinatol. 23(8):679-683. doi:10.1038/sj.jp.7211010 
Nandhini, P., Janney, B., Divakaran, S. 2016. Design of an Intelligent Pillow with Maternal Temperature and Heartbeat Vibrations for Comforting NICU Infants. INDJSRT. 9(33),1-5.

Picciolini, O., Porro, M., Meazza, A., Giannì, M. L., Rivoli, Ch., Lucco,G. 2014. Early exposure to maternal voice: effects on preterm infants development. Early Hum Dev. 90(6), 287-292.

Rand, K., Lahav, A. 2014. Maternal sounds elicit lower heartbeat in preterm newborns in the first month of life. Early Hum Dev. 90(10),679-683.

Saadatmand, V., Rejeh, N., Heravi-Karimooi, M., Tadrisi, S.D., Vaismoradi, M., Jordan, S. 2015. Effects of natural sounds on pain: A randomized controlled trial with patients receiving mechanical ventilation support. Pain Manag Nurs. 16(4), 483-492.

Sanders, M. R., Hall, S. L. 2018. Trauma-informed care in the newborn intensive care unit: promoting safety, security and connectedness. J Perinatol, 38(1), 3-10.

https://doi.org/10.1038/jp.2017.124

Sarhangi, F., Mollahadi, M., Ebadi, A., Matinzadeh, Z.K., Tadrisi, S.D. 2011. Validity and reliability of Neonatal Infant Pain Scale in Neonatal Intensive Care Units in Iran (2010). Pak J Med Sci 27(5):1087-1091.

Suraseranivongse, S., Kaosaard, R., Intakong, P., Pornsiriprasert, S., Karnchana, Y., Kaopinpruck, J., et al. 2006. A comparison of postoperative pain scales in neonates. $\mathrm{Br} \mathrm{J}$ Anaesth. 97(4), 540-544.

WHO Guidelines on Drawing Blood: Best Practices in Phlebotomy. Geneva: World Health Organization; 2010. 6, Paediatric and neonatal blood sampling. Available from: https://www.ncbi.nlm.nih.gov/books/NBK138647/ (Acessed on Juli 8, 2020).

Yildirim, S., Akça, B., Ankay, A., Yilbaş, A., Karagöz, H., Canbay, Ö., Çelebi, N., Öcal, T. 2015. The effects of listening to the mother's heartbeat on the depth of anaesthesia in children Middle East J Anaesthesiol. 23(2),241-246.

Zimmerman, E., Keunen, K., Norton, M., Lahav, A. 2013. Weight gain velocity in very lowbirth-weight infants: effects of exposure to biological maternal sounds. Am J Perinato. 30(10),863-870 

Table 1. Demographic characteristics of the neonates in the groups

\begin{tabular}{|c|c|c|c|c|}
\hline \multicolumn{2}{|l|}{ Variable } & $\begin{array}{l}\text { Control } \\
(\mathbf{n}=30) \\
\text { Mean (SD) }\end{array}$ & $\begin{array}{l}\text { Intervention } \\
(\mathbf{n}=30) \\
\text { Mean(SD) }\end{array}$ & $P$ value and statistics \\
\hline \multicolumn{2}{|l|}{ Age (week) } & $4.43(2.64)$ & $5.23(2.70)$ & $* \mathrm{~F}(58)=023, \mathrm{t}=-1.15, \mathrm{P}=0.25$ \\
\hline \multicolumn{2}{|l|}{ Length (cm) } & $47.50(3.55)$ & $46.60(3.63)$ & $* F(58)=0.18, t=0.97, P=0.33$ \\
\hline \multicolumn{2}{|l|}{ Weight (kg) } & $3.02(0.57)$ & $3.01(0.58)$ & $* F(58)=0.05, t=0.01, P=0.98$ \\
\hline \multicolumn{2}{|c|}{ Gestational age (week) } & $38.07(1.04)$ & 37.97(1.27) & $* * \mathrm{Mdn}=38, \mathrm{u}=436.500, \mathrm{P}=0.83$ \\
\hline \multicolumn{2}{|l|}{ APGAR 1 minute } & $8.97(0.18)$ & $8.87(0.51)$ & $* * \mathrm{Mdn}=9, \mathrm{u}=434, \mathrm{P}=0.53$ \\
\hline \multicolumn{2}{|l|}{ APGAR 5 minute } & $9.97(0.18)$ & $9.80(0.76)$ & $* * \mathrm{Mdn}=10, \mathrm{u}=434, \mathrm{P}=0.53$ \\
\hline & & $n(\%)(n=30)$ & $n(\%)(n=30)$ & $P$ value and statistics \\
\hline \multirow[t]{2}{*}{ Gender } & Male & $14(46.7)$ & $13(43.3)$ & \multirow[t]{2}{*}{ Fisher's Exact Test $=0.99$} \\
\hline & Female & 16(53.3) & $17(56.7)$ & \\
\hline \multirow[t]{2}{*}{ Delivery method } & NVD & $4(13.3)$ & $6(20)$ & \multirow[t]{2}{*}{ Fisher's Exact Test $=0.77$} \\
\hline & $\mathrm{C} / \mathrm{S}$ & $26(86.7)$ & $24(80)$ & \\
\hline
\end{tabular}

APGAR: appearance, pulse, grimace, activity, and respiration; NVD: normal vaginal delivery; C/S: cesarean section

* $\mathrm{P}$ value is calculated by independent t-test and the Fisher's Exact tests for between group comparisons. Kolmogorov-Smirnov P $>0.5$

** $\mathrm{P}$ value is calculated by Mann-Whitney $\mathrm{U}$ test for between group comparisons. Kolmogorov-Smirnov $\mathrm{P}<0.5$ 
Tabel 2. Comparison of hemodynamic parameters before the intervention and at the two follow-ups in the groups

\begin{tabular}{|c|c|c|c|c|}
\hline Group & $\begin{array}{l}\text { Baseline } \\
\text { Mean (SD) }\end{array}$ & $\begin{array}{l}\text { Immediately } \\
\text { after the } \\
\text { interventions } \\
\text { Mean (SD) }\end{array}$ & $\begin{array}{l}10 \text { minutes after } \\
\text { the intervention } \\
\text { Mean (SD) }\end{array}$ & **Pvalue and statistics \\
\hline $\begin{array}{l}\text { HR, } \\
\text { Control }(n=30)\end{array}$ & $127.60(14.90)$ & $155.17(14.66)$ & $130.67(20.44)$ & $\begin{array}{l}\text { Pillai's Trace }=0.76, \mathrm{~F}(2,28)=46.67, \mathrm{P}=0.001 \\
\text { Sphericity Assumed, } \mathrm{F}(2,58)=37.12, \mathrm{P}=0.01\end{array}$ \\
\hline $\begin{array}{l}\mathrm{HR}, \\
\text { Intervention } \\
(\mathrm{n}=30)\end{array}$ & $127.13(12.38)$ & $134.07(12.28)$ & 127.63(9.30) & $\begin{array}{l}\text { Pillai's Trace }=0.26, \mathrm{~F}(2,28)=4.95, \mathrm{P}=0.04 \\
\text { Sphericity Assumed, } \mathrm{F}(2,58)=5.97, \mathrm{P}=0.004\end{array}$ \\
\hline $\begin{array}{l}* \mathbf{P} \text { value and } \\
\text { statistics }\end{array}$ & $\begin{array}{l}F(58)=1.29 \\
t=0.13, P=0.89\end{array}$ & $\begin{array}{l}\mathrm{F}(58)=0.24, \\
\mathrm{t}=6.04, \mathrm{P}=0.001 \\
\text { Cohen's } \mathrm{d}=1.56 \text {, } \\
\text { large. } \text { Effect }\end{array}$ & $\begin{array}{l}F(40.51)=15.007 \\
t=0.74, P=0.46\end{array}$ & $\begin{array}{l}\text { Pillai's Trace }=0.61, \mathrm{~F}(2,57)=46.03, \mathrm{P}=0.001 \\
\text { Sphericity Assumed, } \mathrm{F}(2,116)=41.59, \mathrm{P}=0.01\end{array}$ \\
\hline $\begin{array}{l}\text { RR, Control } \\
(n=30)\end{array}$ & $42.37(12.36)$ & $58.10(14.85)$ & $60.30(14.82)$ & $\begin{array}{l}\text { Pillai's Trace }=0.58, \mathrm{~F}(2,28)=19.58, \mathrm{P}=0.001 \\
\text { Sphericity Assumed, } \mathrm{F}(2,58)=25.25, \mathrm{P}=0.001\end{array}$ \\
\hline $\begin{array}{l}\text { RR, Intervention } \\
(\mathrm{n}=30)\end{array}$ & $44.23(10.05)$ & $50.03(9.41)$ & $49.50(9.64)$ & $\begin{array}{l}\text { Pillai's Trace }=0.26, \mathrm{~F}(2,28)=5.09, \mathrm{P}=0.01 \\
\text { Sphericity Assumed, } \mathrm{F}(2,58)=7.40, \mathrm{P}=0.001\end{array}$ \\
\hline $\begin{array}{l}* P \text { value and } \\
\text { statistics }\end{array}$ & $\begin{array}{l}F(58)=0.37 \\
t=-0.64, P=0.52\end{array}$ & $\begin{array}{l}\mathrm{F}(49.09)=7.39 \\
\mathrm{t}=2.51, \mathrm{P}=0.01 \\
\text { Cohen's d=0.64, } \\
\text { Medium. Effect }\end{array}$ & $\begin{array}{l}\mathrm{F}(49.81)=7.03, \\
\mathrm{t}=3.34, \mathrm{P}=0.001 \\
\text { Cohen's d=0.86, } \\
\text { Large. Effect }\end{array}$ & $\begin{array}{l}\text { Pillai's Trace }=0.46, \mathrm{~F}(2,57)=28.40, \mathrm{P}=0.001 \\
\text { Greenhouse.Geisser, } \mathrm{F}(2,116)=32.35, \mathrm{P}=0.01\end{array}$ \\
\hline $\begin{array}{l}\text { SaO2, Control } \\
(\mathrm{n}=30)\end{array}$ & $91.27(5.54)$ & $87.23(4.79)$ & $90.80(2.67)$ & $\begin{array}{l}\text { Pillai's Trace }=0.27, \mathrm{~F}(2,28)=5.30, \mathrm{P}=0.01 \\
\text { Sphericity Assumed, } \mathrm{F}(2,58)=5.48, \mathrm{P}=0.007\end{array}$ \\
\hline $\begin{array}{l}\mathrm{SaO} 2, \\
\text { Intervention } \\
(\mathrm{n}=30)\end{array}$ & $93.60(3.65)$ & $92.43(4.06)$ & $95.13(2.46)$ & $\begin{array}{l}\text { Pillai's Trace }=0.33, \mathrm{~F}(2,28)=6.89, \mathrm{P}=0.004 \\
\text { Greenhouse.Geisser, } \mathrm{F}(2,58)=6.02, \mathrm{P}=0.007\end{array}$ \\
\hline $\begin{array}{l}\text { * } P \text { value and } \\
\text { statistics }\end{array}$ & $\begin{array}{l}F(58)=0.71 \\
t=-1.92, p=0.059\end{array}$ & $\begin{array}{l}\mathrm{F}(58)=0.23, \\
\mathrm{t}=-4.52, \mathrm{p}=0.001 \\
\text { Cohen's d=1.16, } \\
\text { Large. Effect }\end{array}$ & $\begin{array}{l}\mathrm{F}(39.51)=11.19, \\
\mathrm{t}=-3.83, \mathrm{p}=0.001 \\
\text { Cohen's d=0.98, } \\
\text { Large. Effect }\end{array}$ & $\begin{array}{l}\text { Pillai's Trace }=0.22, \mathrm{~F}(2,57)=8.47, \mathrm{P}=0.01 \\
\text { Sphericity Assumed, } \mathrm{F}(2,116)=9.42, \mathrm{P}=0.001\end{array}$ \\
\hline $\begin{array}{l}\text { MAP, Control } \\
(\mathrm{n}=30)\end{array}$ & $57.71(15.01)$ & $56.73(15.40)$ & 62.32(12.12) & $\begin{array}{l}\text { Pillai's Trace }=0.09, \mathrm{~F}(2,28)=1.54, \mathrm{P}=0.23 \\
\text { Sphericity Assumed, } \mathrm{F}(2,58)=1.60, \mathrm{P}=0.20\end{array}$ \\
\hline $\begin{array}{l}\text { MAP, } \\
\text { Intervention } \\
(n=30)\end{array}$ & 61.32(15.76) & 61.11(16.73) & $60.06(15.38)$ & $\begin{array}{l}\text { Pillai's Trace }=0.008, \mathrm{~F}(2,28)=0.11, \mathrm{P}=0.89 \\
\text { Greenhouse.Geisser, } \mathrm{F}(2,58)=0.07, \mathrm{P}=0.93\end{array}$ \\
\hline $\begin{array}{l}* P \text { value and } \\
\text { statistics }\end{array}$ & $\begin{array}{l}F(58)=0.12 \\
t=-0.90, P=0.36\end{array}$ & $\begin{array}{l}F(25)=0.33 \\
t=-1.05, P=0.29\end{array}$ & $\begin{array}{l}F(58)=2.41 \\
t=0.63, P=0.53\end{array}$ & $\begin{array}{l}\text { Pillai's Trace }=0.01, \mathrm{~F}(2,57)=0.42, \mathrm{P}=0.65 \\
\text { Sphericity Assumed, } \mathrm{F}(2,116)=0.46, \mathrm{P}=0.63\end{array}$ \\
\hline
\end{tabular}

HR: heartbeat; RR: respiratory rate; SaO2: O2 saturation; MAP: mean artrial pressure;

* $\mathrm{P}$ value is calculated by independent t-test for between group comparisons. Kolmogorov-Smirnov $\mathrm{P}>0.5$

**P value is calculated by repeated measure ANOVA test for within group comparisons. Kolmogorov-Smirnov $\mathrm{P}>0.5$

Repeated measure ANOVA $f=\sqrt{\frac{\mathrm{r}^{2}}{1-\mathrm{n}^{2}}}, \mathrm{~d}=2 * f$, Independent t-test $d=\frac{2 t}{\sqrt{d f}}, \quad r=\frac{\sqrt{t^{2}}}{\sqrt{t^{2+d f}}}$ 
Tabel 3. Comparison of mean score of pain before the intervention and at the two follow-ups in the groups

\begin{tabular}{|c|c|c|c|c|}
\hline Group & $\begin{array}{l}\text { Baseline } \\
\text { Mean (SD) }\end{array}$ & $\begin{array}{l}\text { Immediately } \\
\text { after intervention } \\
\text { Mean (SD) }\end{array}$ & $\begin{array}{l}10 \text { minutes after } \\
\text { the Intervention } \\
\text { Mean (SD) }\end{array}$ & $* * \mathbf{P}$ value and statistics \\
\hline $\begin{array}{l}\text { Pain, } \\
\text { Control }(n=30)\end{array}$ & $\begin{array}{l}0.33(54) \\
\text { mild to no pain }\end{array}$ & $\begin{array}{l}5.20(1.06) \\
\text { severe pain }\end{array}$ & $\begin{array}{l}5(1.08) \\
\text { severe pain }\end{array}$ & $\chi^{2}(2,58)=47.43, P=0.001$ \\
\hline $\begin{array}{l}\text { Pain, Intervention } \\
(\mathrm{n}=30)\end{array}$ & $\begin{array}{l}0.20(0.40) \\
\text { mild to no pain }\end{array}$ & $\begin{array}{l}3.97(1.67) \\
\text { mild to moderate pain }\end{array}$ & $\begin{array}{l}3.07(1.68) \\
\text { mild to moderate } \\
\text { pain }\end{array}$ & $\chi^{2}(2,58)=52.41, \mathrm{P}=0.001$ \\
\hline $\begin{array}{l}* P \text { value and } \\
\text { statistics }\end{array}$ & $\begin{array}{l}\mathrm{Mdn}=0, \mathrm{Z}=-0.94 \\
\mathrm{U}=402, \mathrm{P}=0.34\end{array}$ & $\begin{array}{l}\text { Mdn=5,Z=-2.91 } \\
\text { U=260.500, } P=0.004 \\
\text { Cohen's d=0.77, } \\
\text { Medium. Effect }\end{array}$ & $\begin{array}{l}\mathrm{Mdn}=4, \mathrm{Z}=-4.29 \\
\mathrm{U}=166, \mathrm{P}=0.001 \\
\text { Cohen’s d=1.29, } \\
\text { Large. Effect }\end{array}$ & $\chi^{2}(2,118)=92.88, \mathrm{P}=0.001$ \\
\hline
\end{tabular}

* $\mathrm{P}$ value is calculated by Mann-Whitney $\mathrm{U}$ test for between group comparisons. Kolmogorov-Smirnov $\mathrm{P}<0.5$

**P value is calculated by Friedman test for within group comparisons. Kolmogorov-Smirnov $\mathrm{P}<0.5$

Mann-Whitney U $r=\frac{z}{\sqrt{N}}, \quad d=\frac{2 \& r}{\sqrt{1-r^{2}}}$ 


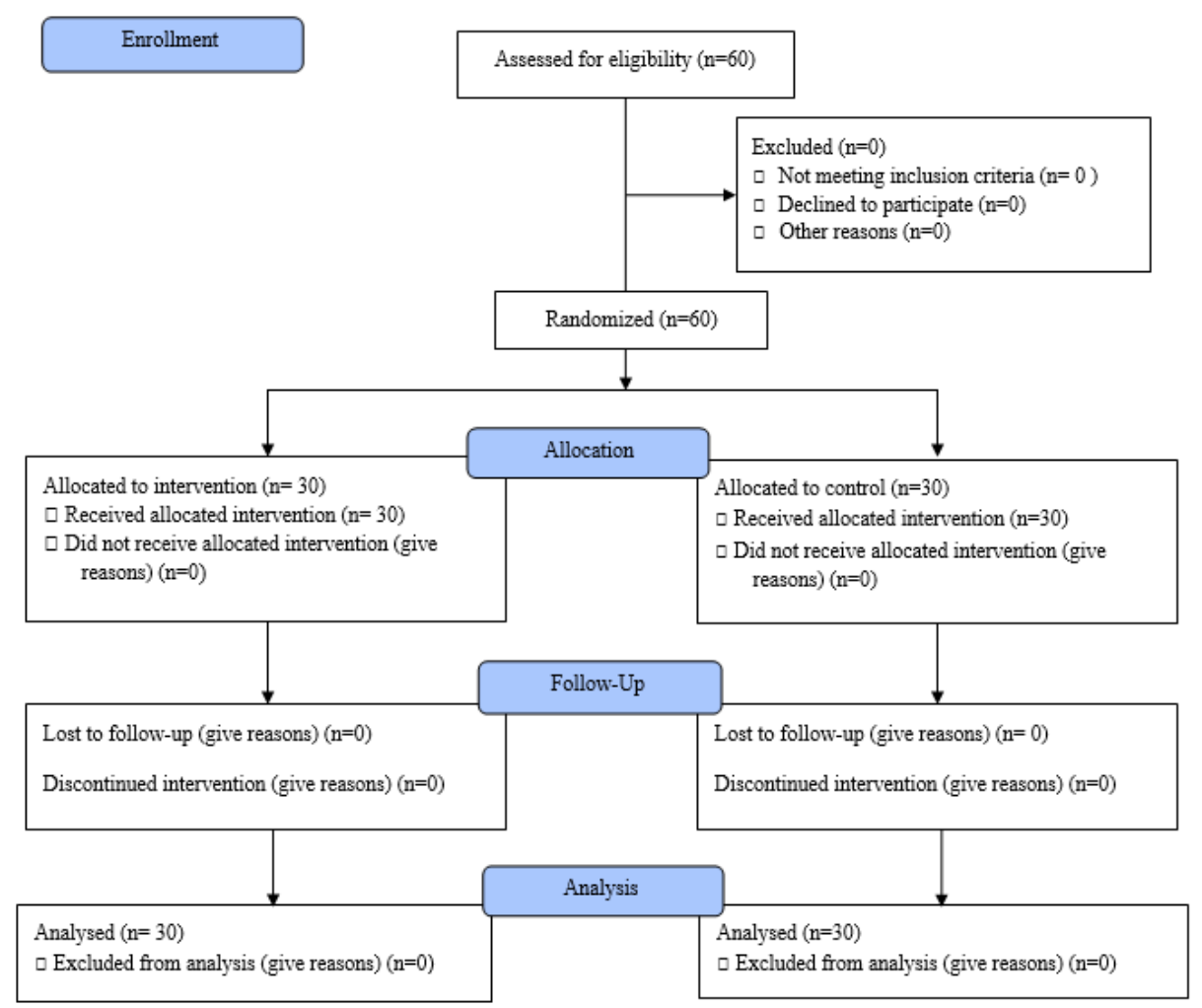

Figure 1. The process of study according to the CONSORT flow diagram 